\title{
Retrospective Review
}

\section{e The Long-Term Outcome of 3-Dimensional CT-Guided Percutaneous Radiofrequency Thermocoagulation for Tumor-Related Trigeminal Neuralgia}

Chen Zheng, MD', Jia Yitong, MD', Jia Zipu, MD1', Wang Tao, MD², and Luo Fang, $\mathrm{MD}^{3}$

From: ${ }^{1}$ Department of Anesthesiology and Pain Management, Beijing Tiantan Hospital, Capital Medical University, Beijing, PR China; 2Department of Neurosurgery, Beijing Tiantan Hospital, Capital Medical University, Beijing, PR China; '3Department of Pain Management, Beijing Tiantan Hospital, Capital Medical University, Beijing, PR China

Address Correspondence: Luo Fang, MD

Department of Pain Management Beijing Tiantan Hospital Capital Medical University Beijing 100070, PR China E-mail: 13611326978@163.com

Disclaimer: This study was supported by the Beijing Municipal Science \& Technology Commission (No. XMLX201707) and the HighLevel Technical Personnel Training Program of the Beijing Municipal Health System (2011-3-034). Both grants equally contributed to the clinical study, data analysis, and manuscript writing. Chen Zheng, Jia Yitong, Jia Zipu, and Wang Tao contributed equally to this work. Conflict of interest: Each author certifies that he or she, or a member of his or her immediate family, has no commercial association (i.e., consultancies, stock ownership, equity interest, patent/licensing arrangements, etc.) that might pose a conflict of interest in connection with the submitted manuscript.

Manuscript received: 12-29-2018 Revised manuscript received: 02-15-2019 Accepted for publication: 03-26-2019

Free full manuscript: www.painphysicianjournal.com
Background: Percutaneous radiofrequency thermocoagulation (PRFT) has been widely used to treat trigeminal neuralgia. By querying MEDLINE, EMBASE, and the Cochrane Library, no study has reported the long-term outcome of PRFT for tumor-related trigeminal neuralgia (TRTN).

Objectives: In this study, we aimed to evaluate the long-term efficacy and safety of PRFT as an alternative treatment for TRTN.

Study Design: A retrospective study.

Setting: The interventional pain management center in Beijing Tiantan hospital.

Methods: We retrospectively analyzed data of all patients who underwent PRFT applied to the Gasserian ganglion under computed tomography guidance for TRTN through a combination of available institutional electronic medical records, patient notes, and radiologic images.

Results: Among 38 patients with PRFT treated between March 2007 and February 2018, 13 patients were men and 25 were women. All patients were evaluated as modified Barrow Neurological Institute (BNI) IV-V before the operation and had a total symptom duration of $45.55 \pm 23.31$ months. The mean operation duration was $59.63 \pm 16.89$ minutes. All patients experienced satisfactory pain relief defined as a classification of BNI I-IIIb within 3 days after PRFT. The median remission length with satisfactory pain relief was 33 (range, 4-132) months. No serious intraoperative complications, except bradycardia in 6 patients, were recorded. Postprocedure complications, including masticatory muscle weakness, were reported in 5 patients. Although all 38 patients experienced facial dysesthesia, the patients Likert scale rating represented that quality of life significantly increased after the procedure.

Limitations: The small sample size may have unavoidably caused selection bias in our study. Larger prospective, randomized, multicenter trials are necessary to validate our outcomes.

Conclusions: PRFT is an effective and safe treatment that should be considered as an alternative for pain control in the treatment of TRTN.

Key words: Pain, secondary trigeminal neuralgia, radiofrequency thermocoagulation, trigeminal neuralgia

Pain Physician 2019: 22:E467-E475-+ 
$\mathrm{T}$ rigeminal neuralgia (TN) is a neuropathic syndrome characterized by lancinating facial pain along the areas innervated by one or more divisions of the trigeminal nerve, which is associated with significant overall reduced quality of life. Approximately $1 \%$ to $13 \%$ of TN cases are secondary to intracranial tumors, and this condition is referred to as tumor-related trigeminal neuralgia (TRTN) $(1,2)$. TRTN can result from a lesion affecting any location along the pathway of the trigeminal nerve (2), such as Meckel's cave (3), the posterior fossa (4), and the cerebellopontine angle (5). The posterior fossa is the most common location of TRTN (4), and meningioma $(4,6)$ and epidermoid cysts $(6,7)$ are the most common pathologies.

The goals of TRTN treatment include controlling tumors and relieving pain (1). Tumor resection is regarded as the most effective treatment, and complete tumor removal is often followed by complete pain remission (4). However, for patients who cannot undergo the risk of a craniotomy and those with end-stage of malignant tumors or slow-growing benign tumors, surgery may not be the best choice. Conservatively, antiepileptics like carbamazepine and oxcarbazepine are considered the first-line therapy for relieving pain (8). However, pharmacology cannot provide satisfactory relief for all patients, and some patients may experience intolerable side effects. For these patients, minimally invasive interventional techniques including percutaneous radiofrequency thermocoagulation (PRFT), percutaneous balloon compression, and percutaneous glycerol rhizolysis are preferred. Radiosurgery has been commonly performed for the treatment of TRTN with promising efficacy $(1,2,9)$. However, this intervention is associated with delayed latency of response $(1,9)$.

PRFT is a low-risk, minimally invasive technique $(10,11)$ with an acute success rate of $97.6 \%$ to $99 \%$ $(11,12)$. PRFT remains the most common percutaneous treatment for TN (13). In addition, this popular procedure may also safely and effectively be repeated if pain recurs (11). The PRFT procedure involves positioning the radiofrequency ( $\mathrm{RF}$ ) needle at the Gasserian ganglion via the foremen ovale (14). Inaccurate piercing may lead to unpleasant complications, including cerebrospinal fluid leakage, cranial nerve (VI) palsy, and intracranial hemorrhage (11). Recently, computed tomography (CT) has been demonstrated to be a useful method to identify the optimal position and predict the intraoperative difficulties of the intervention (15). Nevertheless, no study has reported the long-term outcome of PRFT for TRTN. In this study, we retrospectively analyzed a series of patients who underwent CT-guided PRFT and evaluated the long-term efficacy and safety of PRFT as an alternative treatment for TRTN.

\section{Methods}

\section{Patients}

This retrospective study analyzed patients who underwent PRFT at the Pain Management Center of Beijing Tiantan Hospital attached to Capital Medical University between March 2007 and February 2018. Eligible patients fulfilled the following criteria, aged $>18$ years, typical TN symptoms, brain magnetic resonance imaging confirmed brain tumors that related to $\mathrm{TN}$, trigeminal pain in the same lateral as intracranial tumors, uncontrolled symptoms or intolerable side effects of medication, and pain duration $>90$ days. Notably, patients with other causes of TN, bilateral TN, or a history of mental disorders (schizophrenia and bipolar disorder), or anesthetic drug (sedatives and opioids) abuse were excluded.

\section{Procedures}

All procedures were performed by 2 experienced pain physicians (L.F. and S.Y.) in CT rooms under standard sedation $(0.75 \mathrm{mg} / \mathrm{kg}$ of propofol). After sterilization and local anesthesia, a 21-gauge straight insulated trocar with a 5-mm bare tip (PMF-21-100-5, Baylis Medical Inc., Montreal, Quebec, Canada) was inserted following Hartel's approach. The entry site was $2.5 \mathrm{~cm}$ from the angle of the mouth, and the needle was inserted toward $3 \mathrm{~cm}$ in front of the tragus (sagittal view) and a point below the pupil (coronal view). The CT scanning was performed until the tip of the RF trocar was positioned at Meckel's cave. The tip position was then adjusted to the foremen ovale via guidance by thin-slice scanning, and a 3-dimensional reconstruction was used to verify the location (Fig. $1 \mathrm{~A}$ and B). Optimum position was confirmed by the induction of piercing face pain and jaw movement via sensory and motor stimulation of 0.1 to $0.3 \mathrm{~V}$ at $50 \mathrm{~Hz}$ and $2 \mathrm{~Hz}$, respectively. RF lesioning at the Gasserian ganglion was performed at $60^{\circ} \mathrm{C}$, $65^{\circ} \mathrm{C}, 70^{\circ} \mathrm{C}, 75^{\circ} \mathrm{C}$, and $80^{\circ} \mathrm{C}$ for 75 seconds.

\section{Data Acquisition}

Approval was obtained by the regional ethics committee (KY 2018-024-02) before data collection. Because of the retrospective nature, informed content 
Fig.1. A. In (a) and (b), flat CT scanning of cannula entering the foramen ovale in coronal and sagittal view of skull window. In (c) and (d), flat CT scanning of cannula entering the foramen ovale in coronal and sagittal view of brain window. White marks indicate the cannula, black marks indicate an intracranial meningioma.

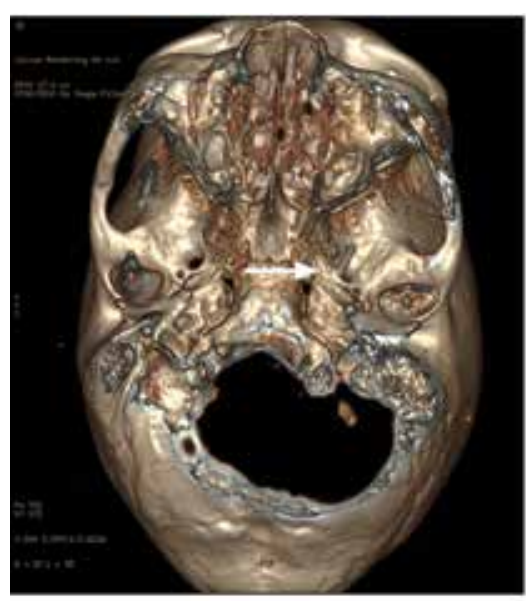

a

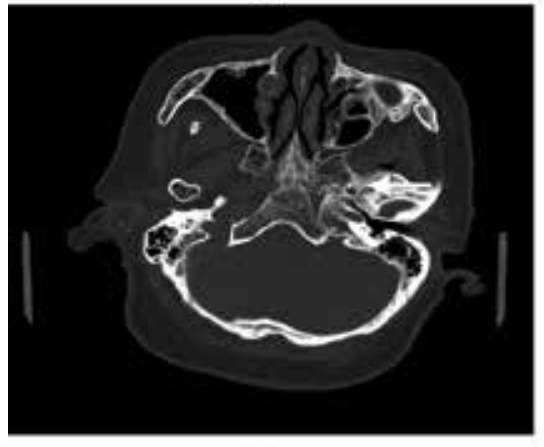

C

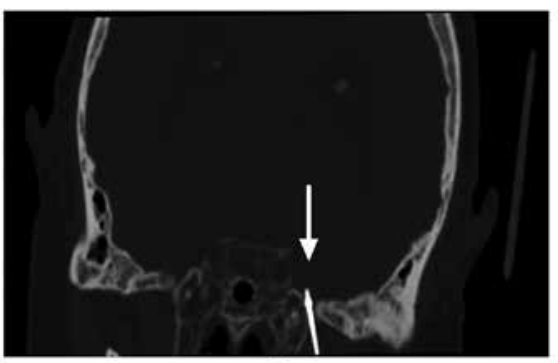

b

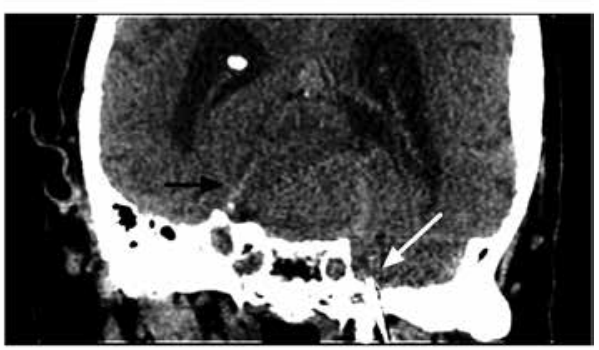

d

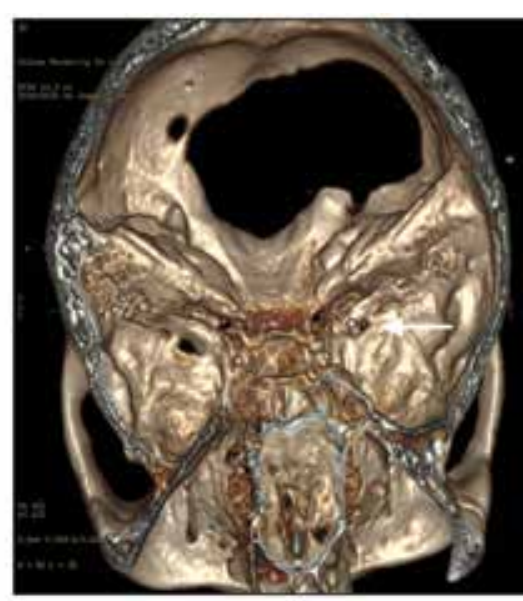

b

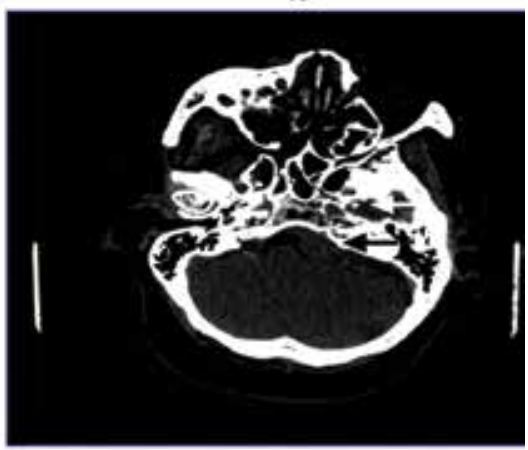

d

B. In (a), threedimensional CT reconstruction image of the cannula entering the foramen ovale in skull base view. In (b), three-dimensional CT reconstruction image of the cannula entering the foramen ovale in interior skull base view. In (c) and (d), scanning of cannula entering the foramen ovale in transverse view. White marks indicate the cannula, black marks indicate an intracranial meningioma. 
was waived. In this study, data were obtained through a combination of available institutional electronic medical records, patient notes, and radiologic images. Follow-up data were acquired by reviewing the follow-up clinic notes that were updated routinely and regularly for clinical practice previously. Patient characteristics were collected for age, gender, laterality, branch affected, comorbidities, preoperative pain intensity, symptom duration, tumor pathology and location, preexisting associated symptoms, and trigger stimuli prior treatment. Operation duration and intraoperative complications were collected for intraoperative issues.

\section{Definitions}

The modified Barrow Neurological Institute (BNI) pain intensity criteria (Table 1) were used to evaluate the pain intensity before and after the operation. Satisfactory pain relief was defined as BNI I-IIIb, and complete pain relief was defined as BNI I after the operation. Initial pain relief was defined as the first satisfactory pain relief after PRFT. A BNI grade that increased to IV-V from I-IIIb after PRFT was defined as recurrence. A BNI grade that never reached I-IIIb was defined as failure. Intraoperative complications included bradycardia and hematoma. Other complications included postoperative nausea and/or vomiting, corneal anesthesia or lost corneal reflex, blindness, facial dysesthesia, masseter weakness, cerebrospinal fluid leakage, carotid cavernous fistula, and perioperative death. A Likert scale (Table 2) was used to grade patients' overall satisfaction.

Table 1. Modified Barrow Neurological Institute pain intensity criteria.

\begin{tabular}{|c|c|}
\hline \hline BNI degrees & Explanations \\
\hline BNI I & No pain, no medication \\
\hline BNI II & Occasional pain, not requiring medication \\
\hline BNI IIIa & No pain with medication \\
\hline BNI IIIb & Controlled pain with medication \\
\hline BNI IV & Improved pain that is inadequately controlled by medication \\
\hline BNI V & Persistent pain that is inadequately controlled by medication \\
\hline
\end{tabular}

Table 2. Likert scale.

\begin{tabular}{|c|c|}
\hline Likert scale & Explanations \\
\hline 1 & Very unsatisfactory \\
\hline 2 & Unsatisfactory \\
\hline 3 & Neutral \\
\hline 4 & Satisfactory \\
\hline 5 & Very satisfactory \\
\hline
\end{tabular}

\section{Statistical Analyses}

Statistical analyses were performed using SPSS Version 20.0 (IBM Corporation, Armonk, NY). All results are reported as the mean \pm standard deviation, median (range), or number. The Kolmogorov-Smirnov test was used to assess the normality of distributions. Data with a normal distribution were compared using independent sample t tests, and the Wilcoxon rank-sum test was used to assess nonparametric variables. Chi-square tests were used to compare categorical data. Statistical significance was indicated by $P<0.05$.

\section{RESULTS}

\section{Demographics}

There were 38 consecutive patients (25 women and 13 men) who underwent CT-guided PRFT for the treatment of TRTN between March 2007 and February 2018. The follow-up time ranged from 4 to 132 months, with a median length of 19.5 months. The patients' demographic and clinical data are described in Table 3. The average age of the patients was $55.61 \pm 16.11$ years. In total, 24 of 38 patients exhibited symptoms on the right side. The morbidity rates of V1, V2, and V3 in TN patients were 3 of 38,7 of 38, and 15 of 38 , respectively. In total, 7 of 38 patients experienced pain in the division of $\mathrm{V} 2+\mathrm{V} 3$. Moreover, 6 of 38 were affected by 3 branches (V1 $+\mathrm{V} 2+\mathrm{V} 3)$ of the TN. Prior treatment included tumor resection (23 of 38), pulsed RF (2 of 38), or both (1 of 38). Headache was associated with TN in 6 patients, whereas nausea was noted in 5 patients. Among the 38 patients, 7 had atrial fibrillation, 5 had diabetes, 10 had hypertension, and 3 had liver carcinoma. Twenty-nine patients had tumors in the posterior fossa, and 9 had tumors in the middle fossa. Tumor pathology included cholesteatoma (13 of 38), meningioma (15 of 38), neurogenic tumors (7 of 38 ), acoustic neuroma (2 of 38), and lipoma (1 of 38). Most patients previously used oral medication, and drug tolerance and severe side effects occurred over time. During the follow-up period, 2 patients were lost at the 32nd and 28th months, owing to lack of telephone contact. 


\section{Pain Relief and Recurrence}

The pain intensity before and after the operation is presented in Table 4. Before PRFT, 26 and 12 patients were classified as BNI IV and V, respectively. The mean symptom duration was $45.55 \pm 23.31$ months. After the intervention, substantial improvement was demonstrated in all the 38 patients. Patients experienced satisfactory pain relief at 33 (range, 4-132) months. All patients obtained satisfactory pain relief 1 day (range, 0-3 days) after the procedure. Among these patients, 1 experienced immediate complete pain relief 2 hours after the operation (BNI I). Twenty-three patients did not experience pain with oral medication (BNI IIla), and 14 gained control of symptoms with anticonvulsant drugs (BNI IIIb). Through the final follow-up visit, satisfactory relief was observed in 21 patients, and 18 patients experienced complete relief. The cumulative recurrence-free survival was presented as a KaplanMeier actuarial curve in Fig. 2. A total of 17 patients experienced relapse of TN symptoms at 18 (range, 6-94) months after PRFT. Within the first year after the procedure, the cumulative recurrence-free survival was $100.0 \%$ at 3 months, $94.4 \%$ at 6 months, and $91.4 \%$ at 12 months. Regarding further outcomes, the cumulative recurrence-free survival was $70.8 \%$ at 24 months, $64.6 \%$ at 36 months, $51.4 \%$ at 60 months, and $40.0 \%$ at 94 to 132 months after the procedure.

\section{Complications}

Intra- and postoperative complications are presented in Table 5. According to a retrospective review of perioperative and clinical data, the mean duration of the procedure was $59.63 \pm 16.89$ minutes. During the operation, 6 patients developed bradycardia when the trocar was adjusted in the foremen ovale. Patients resumed sinus rhythm after interruption of the operation and intravenous injection of $0.01 \mathrm{mg} / \mathrm{kg}$ atropine. None of the patients had corneal anesthesia or ulcer, hematoma, or perioperative death. However, 5 and 4 patients reported masticatory muscle weakness and nausea and/or vomiting, respectively, and all patients developed facial dysesthesia after PRFT. Postoperative nausea and vomiting disappeared within 6 hours after the procedure. Masseter weakness and facial dysesthesia subsided over time. Although these complications were unpleasant, the patients expressed good satisfaction, with a Likert scale rating of 4.0 (3.0-5.0) when initial pain relief was obtained, which was significantly lower than that noted before the procedure (3.0 [1.04.0]; $P<0.05$ ).
Table 3. Patients' demographics.

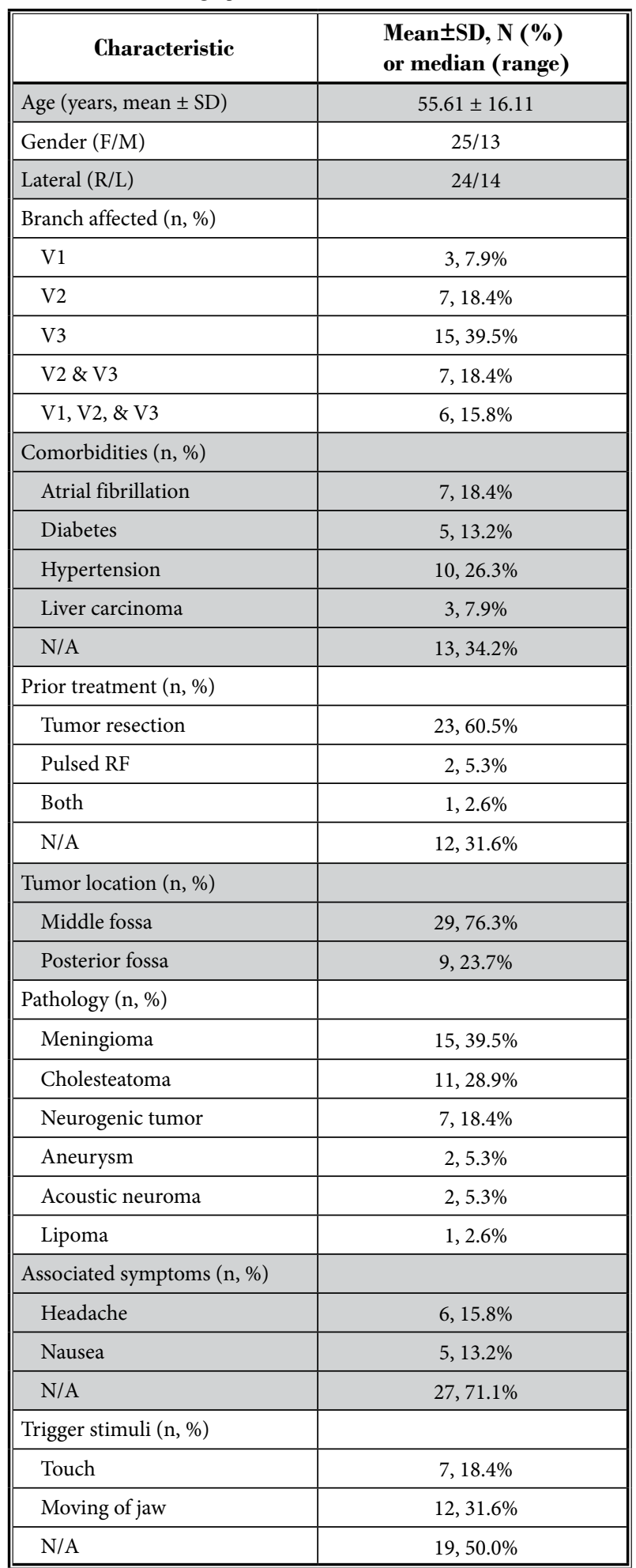

Abbreviations: $F$, female; L, left; M, male; N/A, not available; R, right; $\mathrm{SD}$, standard deviation 


\section{Discussion}

\section{Initial Pain Relief}

Our results revealed that PRFT has a promising initial effect for patients with TRTN. All patients obtained satisfactory pain relief after the procedure. Regarding initial pain relief, $63.2 \%$ of patients did not experience trigeminal

Table 4. Patients' pain intensity pre- and postprocedure.

\begin{tabular}{|l|c|}
\hline Preprocedure (n, \%) & Mean \pm SD, N (\%) or median (range) \\
\hline BNI IV & $26,68.4 \%$ \\
\hline BNI V & $12,31.6 \%$ \\
\hline Symptom duration (months) (mean \pm SD) & $45.55 \pm 23.31$ \\
\hline Initial pain relief (n, \%) & $1,2.6 \%$ \\
\hline BNI I & $23,60.5 \%$ \\
\hline BNI IIIa & $14,36.8 \%$ \\
\hline BNI IIIb & $1(0-3)$ \\
\hline Interval latency (days) [median (range)] & \\
\hline Pain relief at last follow-up (n, \%) & $18,47.4 \%$ \\
\hline BNI I & $2,5.3 \%$ \\
\hline BNI II & $1,2.6 \%$ \\
\hline BNI IIIa & $14,36.8 \%$ \\
\hline BNI IV & $3,7.9 \%$ \\
\hline BNI V & $33(4-132)$ \\
\hline Median maintenance time (months) [median (range)] & \\
\hline
\end{tabular}

Abbreviation: SD, standard deviation.

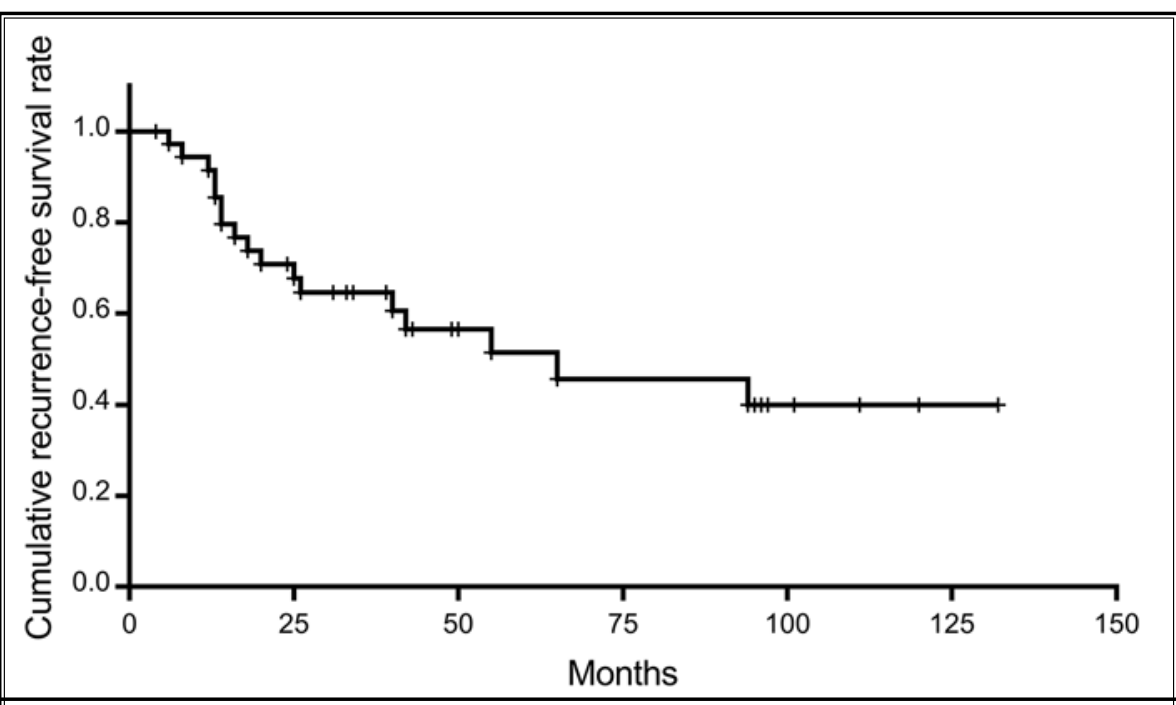

Fig. 2. Kaplan-Meier estimate indicating cumulative recurrence-free survival for 38 patients with TRTN after PRFT. No further pain recurrences occurred after month 94; vertical tick marks indicate follow-up examinations. pain after PRFT with or without the use of oral anticonvulsant drugs (BNII and IIIa), and the remaining $\quad 36.8 \%$ controlled facial pain with medication (BNI 1).The results of our study were similar to those of Kanpolat et al (11) and Fraioli et al (12), who reported that PRFT had an initial success rate of $97.6 \%$ to $99 \%$ for idiopathic TN. Radiosurgery is another effective and safe alternative treatment for TRTN. Studies have demonstrated that radiosurgery had an initial response rate of $81 \%$ to $96 \%$ in the treatment of TRTN $(2,9,16)$. The initial success rate of $100 \%$ for PRFT observed in this study was comparably better than that of radiosurgery. The high acute success rate of PRFT for TRTN currently observed might be explained because the percutaneous approach was guided by CT scanning. Previous studies have suggested that $\mathrm{CT}$ guidance may provide an accurate position for the approach for access to the Gasserian ganglion $(14,17)$. The enhanced puncture accuracy may be responsible for the increased rate of the initial effect.

\section{Long-Term Outcome and Recurrence}

In the present study, patients experienced satisfactory pain relief until 33 (range, 4-132) months after the PRFT procedure. 
The cumulative recurrence-free survival rates remained at $91.4 \%$ and $64.6 \%$ at 12 and 36 months, respectively, after the treatment, with $7.9 \%$ and $31.6 \%$ patients experiencing recurrent trigeminal pain, respectively. Recently, Kim et al (1) suggested that Gamma Knife surgery that targets both the tumors and trigeminal nerve exhibited actuarial recurrencefree survival rates of $93 \%$ at 12 months and $83 \%$ at 36 months postoperative for TRTN, revealing a more reliable method of pain relief.

Although the precise mechanism of TRTN remains unclear, some authors believe that compression induced by the tumor as well as inflammation are related to the neuropathic pain (18). RF thermocoagulation is a destructive treatment that leads to nerve tissue degeneration and prevents synaptic transmission. However, this intervention cannot remove the pathogen in neuropathic disorders. Gamma Knife surgery that targets both nerve tissue and tumors could block the pain signal and reduce the compression induced by adjacent tumors. This feature might explain the more durable pain relief compared with PRFT, which only blocks the pain signal. We hypothesized that tumor-targeted Gamma Knife surgery may mitigate the pathogen of this neuropathic disorder, potentially leading to increased cumulative recurrence-free survival. Regarding further outcomes, the cumulative recurrence-free survival rates were reduced to $51.4 \%$ at 60 months and $40.0 \%$ at 94 to 132 months after PRFT. Evidence indicates that tumors may change the normal anatomy of neurovascular locations and cause increased neurovascular compression (12). Displaced vessels may cause abnormal electrical activity that results in neuropathic pain $(19,20)$. In our opinion, tumors develop or recur over time, and this feature may be associated with recurrence.

\section{Interval Latency}

Prior to our study, Gamma Knife surgery has been a popular treatment for TN with a promising outcome. The efficacy of Gamma Knife surgery for TN treatment is delayed, with a median interval latency between 10 days and 2.5 months after the procedure (21-23). Recently, Kim et al (1) reported that Gamma Knife surgery for TRTN has a median interval latency of 1 month (range, 1-12 months). In the present study, the latency interval to satisfactory pain relief was reduced compared to radiosurgery. All patients reported satisfactory pain relief in $1(0-3)$ day, and no patients reported development of resistance to the intervention. In our opinion, this short latency to pain remission was related
Table 5. Intra- and postprocedure complications.

\begin{tabular}{|l|c|}
\hline \multicolumn{1}{|c|}{ Characteristics } & Patients' Number (n, \%) \\
\hline Intraprocedure complications \\
\hline Bradycardia & $6,15.8 \%$ \\
\hline Postprocedure complications \\
\hline Masseter weakness & $5,13.2 \%$ \\
\hline Nausea and/or vomiting & $4,10.5 \%$ \\
\hline Dysesthesia & $38,100 \%$ \\
\hline \hline
\end{tabular}

to the therapeutic mechanism of PRFT. The procedure destroys myelinated or unmyelinated transduction fibers via a high temperature $\left(80^{\circ} \mathrm{C}\right)$, which is produced by the RF. Pain signals are blocked by the destruction of transmission fibers. However, no standard temperature was identified for optimal pain relief (24). Some authors believe that increasing the temperature of PRFT may increase its effectiveness; however, an increased risk of adverse effects has been noted (25). It is believed that TN is so lancinating that patients prefer the longlasting numbness to the intense pain (26). Therefore a local temperature of $80^{\circ} \mathrm{C}$ was applied for the RF used in our procedure for improved remission. The quality of life of patients was significantly improved within the short recovery period. We suggest that this intervention is more suitable for those in need of urgent TRTN management.

\section{Complications}

An imprecise approach may result in severe complications, such as monocular blindness (27), cerebrospinal fluid leakage (28), and carotid cavernous fistula (11). In our study, all operations were performed under CT guidance, which helped to access the foramen ovale and avoid potential adverse events. However, we observed severe bradycardia when the foramen ovale was punctured. Meng et al (29) reported the same phenomenon in $15.8 \%$ of patients who underwent PRFT for idiopathic TN. Bradycardia during the percutaneous procedure was considered a trigeminal reflex (30), and the operation was interrupted for heart rate recovery (29). Different degrees of dysesthesia occurred after PRFT in $1 \%$ to $8 \%$ of patients with idiopathic TN $(11,31-33)$, and $10.5 \%$ to $21.1 \%$ of patients undergoing radiosurgery $(1,22,23,34)$. All patients in our study experienced facial dysesthesia after the intervention, which was previously observed by Zheng et al (35). We hypothesized that a temperature of $80^{\circ} \mathrm{C}$ at the precise position of the Gasserian ganglion may completely destroy the sensitive fibers. The destruc- 
tion may block both pain signal transduction and normal sensory function. In addition, $13.2 \%$ and $10.5 \%$ of patients reported masseter weakness and nausea and/ or vomiting after the intervention, respectively. All patients with nausea and/or vomiting recovered within 6 hours after PRFT. The intensity of masseter weakness and facial dysesthesia decreased to a tolerable level several months after PRFT. Although these complications may lead to unpleasant experiences, the Likert scale ratings of patients were significantly increased after treatment due to satisfactory pain control. Given the wide use of minimally destructive techniques, such as pulsed RF, we anticipate fewer postprocedure complications for patients. Unfortunately, 2 patients experienced failure of pulsed RF prior to our treatment. The efficacy of pulsed RF applied for TRTN has yet to be investigated in larger clinical trials and with adjusted parameters.

\section{Limitations}

Given its retrospective design, our study has several limitations. The small sample size may have unavoidably caused selection bias in our study. Further investigations on the prevention of intraoperative bradycardia and postoperative nausea and vomiting, facial dysesthesia, and masseter weakness are of great importance. Larger prospective, randomized, multicenter clinical trials are necessary to validate our outcomes.

\section{Conclusions}

To the best of our knowledge, this is the first study to report the long-term outcomes of PRFT for TRTN under CT guidance. The long-term outcomes of our study demonstrate that PRFT is an effective and safe treatment for patients with TRTN. The short latency interval to satisfactory pain relief is the main advantage of this procedure. This technique should be considered as an alternative for pain control in the treatment of TRTN.

\section{References}

1. Kim SK, Kim DG, Se YB, Kim JW, Kim $\mathrm{YH}$, Chung HT, Paek SH. Gamma Knife surgery for tumor-related trigeminal neuralgia: Targeting both the tumor and the trigeminal root exit zone in a single session. J Neurosurg 2016; 125:838-844.

2. Cho KR, Lee MH, Im YS, Kong DS, Seol HJ, Nam DH, Lee Jl. Gamma Knife radiosurgery for trigeminal neuralgia secondary to benign lesions. Headache 2016; 56:883-889.

3. Furtado SV, Hegde AS. Trigeminal neuralgia due to a small Meckel's cave epidermoid tumor: Surgery using an extradural corridor. Skull Base 2009; 19:353-357.

4. Cheng TM, Cascino TL, Onofrio BM. Comprehensive study of diagnosis and treatment of trigeminal neuralgia secondary to tumors. Neurology 1993; 43:2298-2302.

5. Khan N, Michael A, Choucair A, Bit-Ivan E. Trigeminal ganglioneuroma: A rare case of trigeminal neuralgia caused by cerebellopontine angle tumor. World Neurosurg 2017; 99:811.e7-811.e10.

6. Liu P, Liao C, Zhong W, Yang M, Li S, Zhang $W$. Symptomatic trigeminal neuralgia caused by cerebellopontine angle tumors. J Craniofac Surg 2017; 28:e256-e258.
7. Kobata H, Kondo A, Iwasaki K. Cerebellopontine angle epidermoids presenting with cranial nerve hyperactive dysfunction: Pathogenesis and long-term surgical results in 30 patients. Neurosurgery 2002; 50:276-285; discussion 285-276.

8. Huang $Q$, Liu X, Chen J, Bao C, Liu D, Fang Z, Liang X, Lu Z, Wan L. The effectiveness and safety of thermocoagulation radiofrequency treatment of the ophthalmic division ( $V_{1}$ ) and/or maxillary $\left(V_{2}\right)$ and mandibular $\left(V_{3}\right)$ division in idiopathic trigeminal neuralgia: An observational study. Pain Physician 2016; 19:E1041-E1047.

9. Huang CF, Tu HT, Liu WS, Lin LY. Gamma Knife surgery for trigeminal pain caused by benign brain tumors. ] Neurosurg 2008; 109(Suppl):154-159.

10. Nguyen M, Wilkes D. Pulsed radiofrequency $V_{2}$ treatment and intranasal sphenopalatine ganglion block: A combination therapy for atypical trigeminal neuralgia. Pain Pract 2010; 10:370-374.

11. Kanpolat Y, Savas A, Bekar A, Berk C. Percutaneous controlled radiofrequency trigeminal rhizotomy for the treatment of idiopathic trigeminal neuralgia: 25year experience with 1,600 patients. Neurosurgery 2001; 48:524-532; discussion 532-524.
12. Fraioli B, Esposito V, Guidetti B, Cruccu G, Manfredi M. Treatment of trigeminal neuralgia by thermocoagulation, glycerolization, and percutaneous compression of the gasserian ganglion and/ or retrogasserian rootlets: Long-term results and therapeutic protocol. Neurosurgery 1989; 24:239-245.

13. Emril DR, Ho KY. Treatment of trigeminal neuralgia: Role of radiofrequency ablation. J Pain Res 2010; 3:249-254.

14. Easwer HV, Chatterjee N, Thomas A, Santhosh K, Raman KT, Sridhar R. Usefulness of flat detector CT (FD-CT) with biplane fluoroscopy for complication avoidance during radiofrequency thermal rhizotomy for trigeminal neuralgia. J Neurointerv Surg 2016; 8:830-833.

15. Guo Z, Wu B, Du C, Cheng M, Tian Y. Stereotactic approach combined with 3D CT reconstruction for difficult-toaccess foramen ovale on radiofrequency thermocoagulation of the gasserian ganglion for trigeminal neuralgia. Pain Med 2016; 17:1704-1716.

16. Pollock BE, luliano BA, Foote RL, Gorman DA. Stereotactic radiosurgery for tumor-related trigeminal pain. Neurosurgery 2000; 46:576-582; discussion 582-573.

17. Fang L, Ying S, Tao W, Lan M, Xiaotong 
Y, Nan J. 3D CT-guided pulsed radiofrequency treatment for trigeminal neuralgia. Pain Pract 2014; 14:16-21.

18. Bennett GJ. Pathophysiology and animal models of cancer-related painful peripheral neuropathy. Oncologist 2010; 15(Suppl 2):9-12.

19. Barker FG 2nd, Jannetta PJ, Babu RP, Pomonis S, Bissonette DJ, Jho HD. Long-term outcome after operation for trigeminal neuralgia in patients with posterior fossa tumors. J Neurosurg 1996; 84:818-825.

20. Bullitt E, Tew JM, Boyd J. Intracranial tumors in patients with facial pain. J Neurosurg 1986; 64:865-871.

21. Taich ZJ, Goetsch SJ, Monaco E, Carter BS, Ott K, Alksne JF, Chen CC. Stereotactic radiosurgery treatment of trigeminal neuralgia: Clinical outcomes and prognostic factors. World Neurosurg 2016; 90:604-612.e11.

22. Regis J, Tuleasca C, Resseguier N, Carron R, Donnet A, Gaudart J, Levivier M Long-term safety and efficacy of Gamma Knife surgery in classical trigeminal neuralgia: A 497-patient historical cohort study. J Neurosurg 2016; 124:1079-1087.

23. Mousavi SH, Niranjan A, Huang MJ, Laghari FJ, Shin SS, Mindlin JL, Flickinger JC, Lunsford LD. Early radiosurgery provides superior pain relief for trigeminal neuralgia patients. Neurology 2015; 85:2159-2165.
24. Elawamy A, Abdalla EEM, Shehata GA. Effects of pulsed versus conventional versus combined radiofrequency for the treatment of trigeminal neuralgia: A prospective study. Pain Physician 2017; 20:E873-E881.

25. Bogduk N, Macintosh J, Marsland A. Technical limitations to the efficacy of radiofrequency neurotomy for spinal pain. Neurosurgery 1987; 20:529-535.

26. Eugene AR. Trigeminal neuralgia and radiofrequency lesioning. Brain (Bacau) 2015; 6:91-96.

27. Egan RA, Pless M, Shults WT. Monocular blindness as a complication of trigeminal radiofrequency rhizotomy. Am J Ophthalmol 2001; 131:237-240.

28. Ugur HC, Savas A, Elhan A, Kanpolat Y. Unanticipated complication of percutaneous radiofrequency trigeminal rhizotomy: Rhinorrhea: Report of three cases and a cadaver study. Neurosurgery 2004; 54:1522-1524; discussion 1524-1526.

29. Meng $\mathrm{Q}$, Zhang W, Yang $\mathrm{Y}$, Zhou M, Li $X$. Cardiovascular responses during percutaneous radiofrequency thermocoagulation therapy in primary trigeminal neuralgia. J Neurosurg Anesthesiol 2008; 20:131-135

30. Cheng JS, Lim DA, Chang EF, Barbaro NM. A review of percutaneous treatments for trigeminal neuralgia. Neuro- surgery 2014; 10(Suppl 1):25-33; discussion 33 .

31. Scrivani SJ, Keith DA, Mathews ES, Kaban LB. Percutaneous stereotactic differential radiofrequency thermal rhizotomy for the treatment of trigeminal neuralgia. J Oral Maxillofac Surg 1999; 57:104-111; discussion 111-102.

32. Kosugi S, Shiotani M, Otsuka $Y$, Suzuki T, Katori N, Hashiguchi S, Morisaki H. Long-term outcomes of percutaneous radiofrequency thermocoagulation of gasserian ganglion for 2nd- and multiple-division trigeminal neuralgia. Pain Pract 2015; 15:223-228.

33. Son BC, Kim HS, Kim IS, Yang SH, Lee SW. Percutaneous radiofrequency thermocoagulation under fluoroscopic image-guidance for idiopathic trigeminal neuralgia. J Korean Neurosurg Soc 2011; 50:446-452.

34. Kondziolka D, Zorro O, Lobato-Polo J, Kano H, Flannery TJ, Flickinger JC, Lunsford LD. Gamma Knife stereotactic radiosurgery for idiopathic trigeminal neuralgia. J Neurosurg 2010; 112:758-765.

35. Zheng S, Wu B, Zhao Y, Wang X, Li X, Yang L, He M, Yue J, Ni J. Masticatory muscles dysfunction after CT-guided percutaneous trigeminal radiofrequency thermocoagulation for trigeminal neuralgia: A detailed analysis. Pain Pract 2015; 15:712-719. 
